\title{
Open Information Extraction with Meta-pattern Discovery in Biomedical Literature
}

\author{
Xuan Wang \\ University of Illinois at \\ Urbana-Champaign \\ xwang174@illinois.edu
}

\author{
Yu Zhang \\ University of Illinois at \\ Urbana-Champaign \\ yuz9@illinois.edu
}

\author{
Qi Li \\ University of Illinois at \\ Urbana-Champaign \\ qili5@illinois.edu
}

\author{
Yinyin Chen \\ University of Illinois at \\ Urbana-Champaign \\ ychen409@illinois.edu
}

\author{
Jiawei Han \\ University of Illinois at \\ Urbana-Champaign \\ hanj@illinois.edu
}

\begin{abstract}
Biomedical open information extraction (BioOpenIE) is a novel paradigm to automatically extract structured information from unstructured text with no or little supervision. It does not require any pre-specified relation types but aims to extract all the relation tuples from the corpus. A major challenge for open information extraction (OpenIE) is that it produces massive surface-name formed relation tuples that cannot be directly used for downstream applications. We propose a novel framework CPIE (Clause+Pattern-guided Information Extraction) that incorporates clause extraction and metapattern discovery to extract structured relation tuples with little supervision. Compared with previous OpenIE methods, CPIE produces massive but more structured output that can be directly used for downstream applications. We first detect short clauses from input sentences. Then we extract quality textual patterns and perform synonymous pattern grouping to identify relation types. Last, we obtain the corresponding relation tuples by matching each quality pattern in the text. Experiments show that CPIE achieves the highest precision in comparison with state-of-the-art OpenIE baselines, and also keeps the distinctiveness and simplicity of the extracted relation tuples. CPIE shows great potential in effectively dealing with real-world biomedical literature with complicated sentence structures and rich information.
\end{abstract}

\section{CCS CONCEPTS}

- Information systems $\rightarrow$ Data mining; • Applied computing $\rightarrow$ Life and medical sciences;

\section{KEYWORDS}

open information extraction; pattern mining; biomedical information extraction; text mining

Permission to make digital or hard copies of all or part of this work for personal or classroom use is granted without fee provided that copies are not made or distributed for profit or commercial advantage and that copies bear this notice and the full citation on the first page. Copyrights for components of this work owned by others than ACM must be honored. Abstracting with credit is permitted. To copy otherwise, or republish, to post on servers or to redistribute to lists, requires prior specific permission and/or a fee. Request permissions from permissions@acm.org.

ACM-BCB'18, August 29-September 1, 2018, Washington, DC, USA

(C) 2018 Association for Computing Machinery.

ACM ISBN 978-1-4503-5794-4/18/08 ...\$15.00

https://doi.org/10.1145/3233547.3233594

\section{ACM Reference Format:}

Xuan Wang, Yu Zhang, Qi Li, Yinyin Chen, and Jiawei Han. 2018. Open Information Extraction with Meta-pattern Discovery in Biomedical Literature. In ACM-BCB'18: 9th ACM International Conference on Bioinformatics, Computational Biology and Health Informatics, August 29-September 1, 2018, Washington, DC, USA. ACM, New York, NY, USA, 10 pages. https: //doi.org/10.1145/3233547.3233594

\section{INTRODUCTION}

Biomedical information extraction (BioIE) aims to automatically extract structured semantic information from unstructured text. It includes tasks such as named entity recognition, relation extraction, and event detection. BioIE has been successfully applied to many downstream applications such as clinical decision support [12] and question answering [4], biological pathway and network analysis [32], integrative biology [35], biocuration [36, 40, 42] and pharmacovigilance [17]. Machine learning (ML) models are widely adopted in current BioIE systems. For example, in a chemical-induced disease (CID) relation extraction task in the recent BioCreative $\mathrm{V}$ challenge [41], systems are proposed using models such as support vector machine [33], feature kernels [24] and convolutional neural network [15]. However, ML models rely on human annotation for training data generation, which is time and labor consuming. Moreover, the reliance on human annotation further limits the systems to certain pre-specified relation types and makes it unable to further extend the systems to new relation types.

Open information extraction (OpenIE), a novel information extraction paradigm, begins to attract great attention in the BioIE domain. OpenIE does not require any pre-specified relation types but aims to extract all the relation tuples from a corpus with no or little human supervision. Two major types of method are proposed for OpenIE: clause-based methods and pattern-based methods. Clausebased methods use linguistic features or sentence structures to induct long-distance relationships from sentences [2, 10, 13, 37]. For example, ClausIE [10] performs clause type analysis based on dependency parsing, chunking, and POS tagging to extract all the possible clauses from the input sentences [10]. In the "Clause extraction" step in Figure 1, eight different clauses can be extracted from the input sentence by ClausIE. The clause-based method extracts massive subject-verb-object relation tuples from the corpus in the surface-name form. Pattern-based methods exploit entity type 
information and frequent pattern mining to extract entity-attributevalue (EAV) tuples from input sentences [20, 23, 29]. For example, MetaPAD [20] uses context-aware segmentation and synonymous pattern grouping to extract texture meta-patterns and the value tuples from the input sentence [20]. A meta-pattern is a relationship between entity types. In the "Meta-pattern extraction" step in Figure 1, a meta-pattern "CHEMICAL decreased susceptibility to DISEASE in young SPECIES" can be extracted, which is a relation between three entity types. These meta-patterns can further be used to extract corresponding relation tuples from the corpus.

Both types of OpenIE methods have their pros and cons. Clausebased methods are good at resolving long and complicated sentence structures. However, the massive subject-verb-object relation tuples they extract are in purely surface-name form, which cannot be directly used for downstream applications. Pattern-based methods are easy to be extended to any n-ary relations and produce more structured output with entity type information. However, when the whole sentence has a complicated structure, the tokens between the entities can be lengthy, resulting in the sparsity of relation patterns extracted by the pattern-based method. OpenIE methods have been successfully applied in general domain such as extracting information from newspapers. However, they achieved limited success when applying to the biomedical domain due to some specific challenges with the biomedical text:

(1) Sentences are usually long with complicated structures. The entities within one relation may be far apart in a sentence, and one sentence may contain more than one relation type and more than one relation tuple. This greatly limits the performance of current pattern-based methods.

(2) The entity type information is important for both relation type selection/consolidation and relation instance interpretation. This is not considered by clause-based methods.

(3) High-ary relationships exist in sentences, which can provide more complete and accurate information than binary ones. This is also not considered by clause-based methods.

To address the above challenges, we propose a novel framework CPIE: Clause+Pattern-guided Information Extraction as shown in Figure 1. CPIE combines the merits of clause-based and pattern-based methods, extracting both relation types (meta-pattern synonymous groups) and relation tuples. The framework requires no supervision for clause extraction, meta-pattern extraction and relation tuple extraction, only a few positive examples for quality metapattern selection. Compared with previous OpenIE methods, CPIE produces massive but more structured output that can be directly used for downstream applications. We first resolve the long and complicated sentence structures by extracting short clauses from the input sentences. Then we perform meta-pattern extraction on the short clauses. Quality meta-patterns are selected and synonymous meta-patterns are grouped together as a single relation type. Then quality meta-patterns are used to extract relation tuples from the input sentences. Experiments show that our method achieves the highest precision in comparison with state-of-the-art OpenIE baselines, and also keeps the distinctiveness and simplicity of the extracted relation tuples. Case studies also show the power of CPIE in effectively dealing with real-world biomedical literature with complicated sentence structures and rich information.

\section{RELATED WORK}

Open-Domain Information Extraction. OpenIE aims to find new extraction paradigms and extract large sets of relational tuples from a corpus with no or little human supervision. OpenIE has been extensively studied in the NLP area. The task of OpenIE was first introduced by the seminal work of Banko et al. [3]. After their TextRunner system, most of the existing work follows two lines: clause-based methods and pattern-based methods.

For clause-based analysis, linguistic features, like dependency parsing results, are used to induct long-distance relationships. For example, ReVerb [11] identifies relational phrases via part-of-speechbased regular expressions. Ollie [37] further expands the syntactic scope of relation phrases and allows additional context information such as attribution and clausal modifiers. Similarly, ClausIE [10] inducts short but coherent pieces of information along dependency paths, which is typically subject, predicate and optional object with complement. Stanford OpenIE [2] adopts a clause splitter using distant training and mapped predicates to a known relation schema statistically. MinIE [13] further improves the clearness of relation tuples by introducing different statistical measures like polarity, modality, attribution, and quantities. ReMine [44] integrates local context and global cohesiveness to reduce the amount of uninformative or incoherent tuples.

Pattern-based information extraction can be traced back to Hearst patterns, such as " $N P_{0}$ such as $\left\{N P_{1}, N P_{2}, \ldots\right\}$ ", which are used for hyponymy relation extraction [18]. Mitchell et al. [27] introduce Never-Ending Language Learning (NELL) based on free-text predicate patterns. Patty [29] aims to extract a set of typed lexical patterns along the shortest dependency path. MetaPAD [20] generates quality meta-patterns (i.e., relational patterns between entity types) by context-aware segmentation, groups synonymous meta-patterns, and adjusts entity-type levels for appropriate granularity in the pattern groups. TruePIE [23] further adopts truth discovery ideas to extract reliable meta-patterns. Using these patterns to match the corpus, tuples with entities and relation phrases can be extracted.

Biomedical Open Information Extraction. BioIE aims to automatically extract structured semantic information from unstructured biomedical corpus. It includes tasks such as named entity recognition, relation extraction, and event extraction. The methods for BioIE include dictionary-based methods, rule-based methods, statistical methods, classification-based methods and hybrid methods $[39,43]$. Several survey papers are published containing thorough and systematic summaries of previous work on BioIE [1, 5-7, 14, 16, 19, 22, 26, 34, 39, 43, 45]. We focus on introducing some related work about BioOpenIE, which is a novel paradigm of BioIE that is most related to our work.

OpenIE, as a novel information extraction paradigm, begins to attract great attention in BioIE domain. It does not require any prespecified relation types but aims to extract all the relation tuples from a corpus with no or little human supervision. Liu et al. [25] introduce some applications of OpenIE to BioIE in their survey paper. For example, Attias et al. [28] present an OpenIE system for biomedical named entity recognition based on NELL [27], and propose a method for assessing seed qualities to prevent semantic drift. Nebot et al. [30, 31] propose a scalable method to extract surfaceform biomedical relationships not specific to any relation type and 


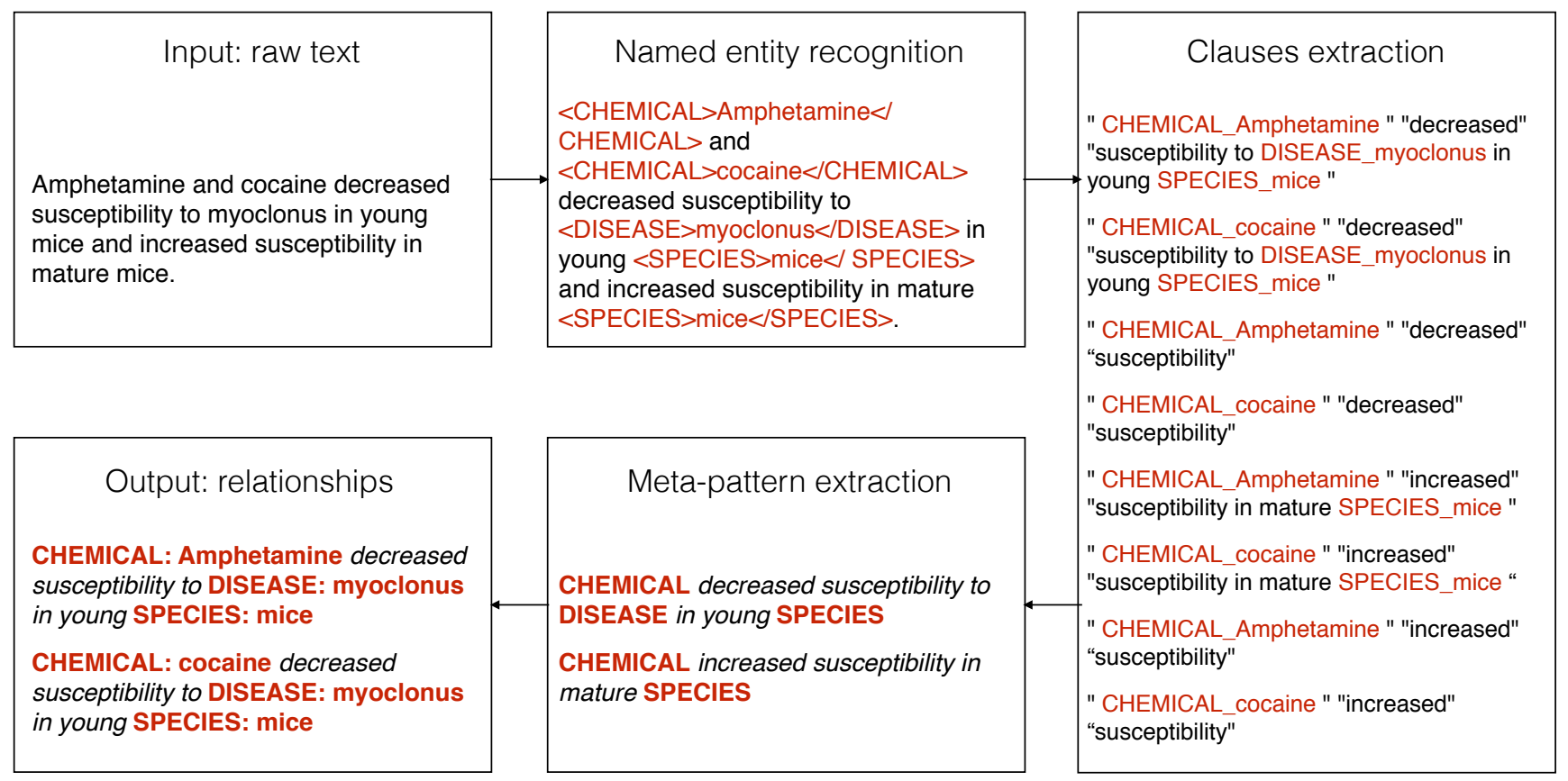

Figure 1: The overall framework of CPIE: Clause+Pattern-guided Information Extraction.

further infer the semantic types of the extracted relationships by clustering. De Silva et al. [9] discover inconsistencies in PubMed abstracts through ontology-based information extraction, in which Ollie [37] is a key step.

\section{THE BIOOPENIE FRAMEWORK}

The overall framework of CPIE is shown in Figure 1. The inputs are sentences from a biomedical corpus. The first step is biomedical named entity recognition (BioNER), which recognizes and classifies the biomedical entities with their proper entity types, e.g., gene, chemical, and disease. Each entity mention is treated as an integrated unit in the process. Then we select the sentences that contain at least one typed entity and perform clause extraction to extract short clauses from the typed sentences. These short clauses are further selected as those that contain at least one typed entity. In each selected clause, we replace the entity mention with its type and extract all the quality meta-patterns as fine-grained relationship types. These quality meta-patterns are further grouped into synonymous groups. Last, we use the quality meta-patterns to extract their corresponding relationship instances from the original input sentences. The extracted meta-patterns and relationship instances are the output of our framework.

Biomedical named entity recognition. We perform BioNER as a preprocessing step of our pipeline to generate a typed corpus. We use Pubtator, a state-of-the-art BioNER tool, to recognize and type the biomedical entities from the corpus [40]. It includes five biomedical entity types: gene/protein, chemical, disease, species, and SNP. Pubtator provides a fully annotated version of the PubMed abstracts that can be directly downloaded from https://www.ncbi. nlm.nih.gov/CBBresearch/Lu/Demo/PubTator/.

\section{CLAUSE EXTRACTION}

As mentioned above, if the whole sentence has a complicated structure, the tokens between the entities can be lengthy, resulting in the sparsity of relation patterns. To alleviate this problem, we first extract clauses, which are simplified and express some coherent piece of information, from the sentence.

Definition 4.1 (Clause Extraction). A clause is a part of a sentence that consists of one subject (S), one verb (V), and optionally of an indirect object $(\mathrm{O})$, a direct object $(\mathrm{O})$, a complement $(\mathrm{C})$, and one or more adverbials (A). The goal of clause extraction is to segment the whole sentence into clauses and represent them in the format of (subject, relation, optional components).

We use ClausIE [10] for clause extraction. We first concatenate all the words in each entity mention with underlines to make them an integrated unit. Then we select those sentences with at least one typed entity as the input for ClausIE.

For each input sentence, ClausIE conducts the following steps: Dependency Parsing. We adopt Stanford dependency parser [21] to discover the syntactical structure of a sentence. The output parsing tree has a set of directed syntactic relations between the words in the sentence. Figure 2 shows an example. The root of the tree is the verb "appear". It is connected to "doses" via a subject relation (nsubj) and to "useful" via a complement relation (xcomp). For a complete list of relations, please refer to https://nlp-ml.io/jg/ software/pac/standep.html.

Clause Identification. According to the dependency parsing tree, ClausIE first constructs a clause for each subject relation (e.g., nsubj) connecting the subject (S) and the governor verb (V). Besides, objects (O) and complements (C) linked with V through dobj, iobj, 
Table 1: Patterns and clause types we use [10]. $S$ : Subject, $V:$ Verb, $C$ : Complement, $O:$ Direct object, $O_{i}$ : Indirect object, $A$ : Adverbial, $V_{i}$ : Intransitive verb, $V_{c}$ : Copular verb, $V_{c}$ : Extended-copular verb, $V_{m t}$ :Monotransitive verb, $V_{d t}:$ Ditransitive verb, $V_{c t}:$ Complex-transitive verb.

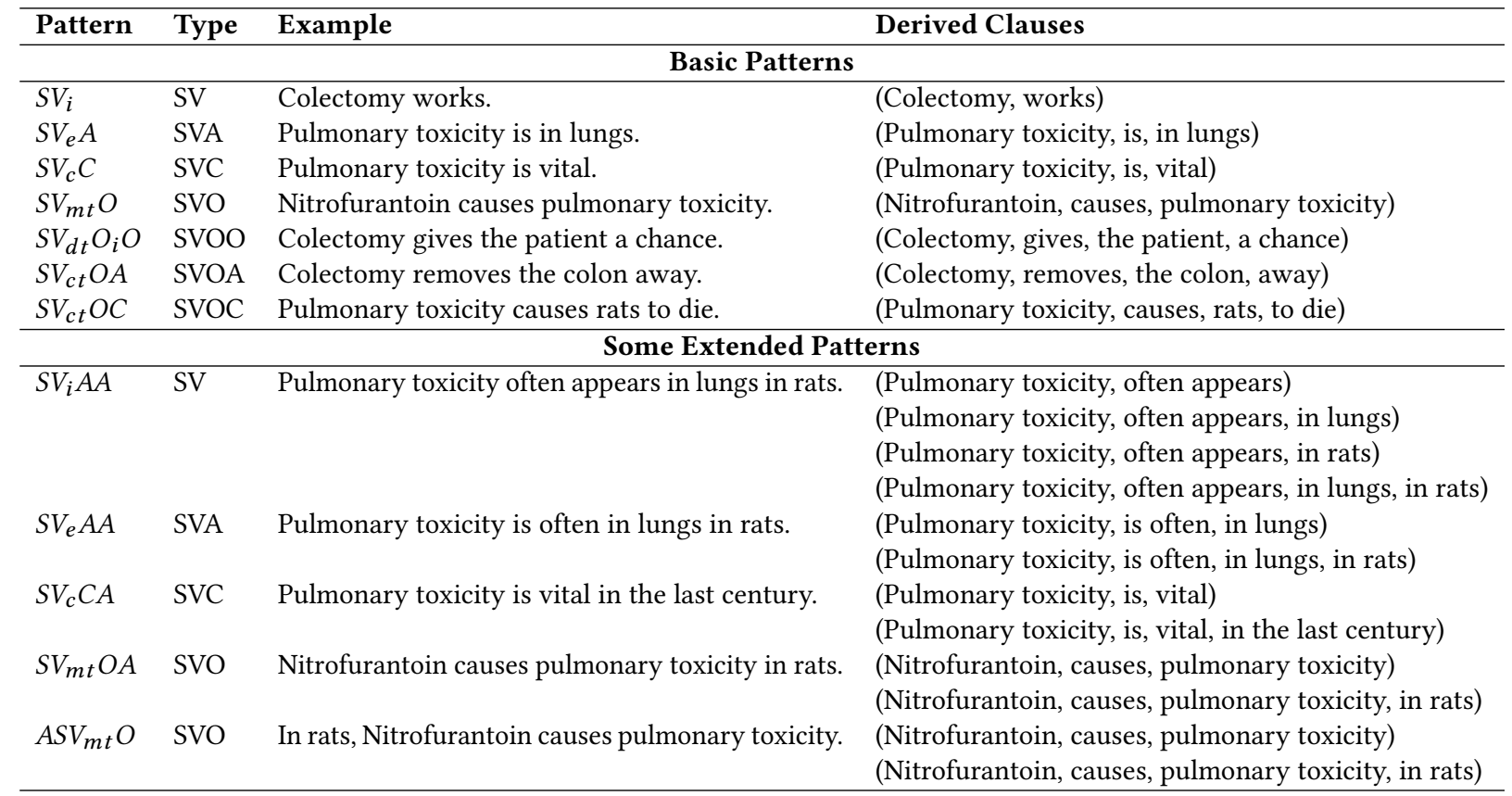

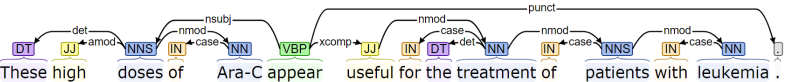

Figure 2: An example sentence with dependency parse.

xcomp or ccomp, as well as adverbials (A) related with $\mathrm{V}$ through advmod, advcl or prep_in will also be included.

Clause Type Analysis. Once clauses have been identified, ClausIE tries to analyze the type of each clause. A complete list of all clause types used in [10] is given in Table 1. Based on the lexical and syntactical structure, the clause type classification is essentially a decision tree. The complete decision flow can be found in [10].

Proposition Generation. Given the clause type, we are able to decide which tokens to place into the subject, the relation, and the optional components. ClausIE maps the subject of the clause to the subject of the proposition. For the optional components, an argument is created and includes the tokens following the verb and then the tokens preceding the verb, in the order in which they appear.

If there is no clause extracted for an input sentence, we will keep the original input sentence as the output clause. The output clauses are further selected to contain at least one typed entity for meta-pattern extraction.

\section{META-PATTERN EXTRACTION}

Definition 5.1 (Meta-pattern Extraction). Given a corpus $C$ as a list of typed sentences (clauses) $S$, i.e., $C=\left[S_{1}, S_{2}, \ldots, S_{n}\right]$, each sentence is a sequence of word tokens $t$, i.e., $S=t_{1} t_{2} \ldots t_{m}$, in which $t_{j} \in \mathcal{T} \cup \mathcal{W}(\mathcal{T}$ is the set of entity types and $\mathcal{W}$ is the set of non-type words). The goal of meta-pattern extraction is to extract meta-patterns $m p$, which are sub-sequences of the typed sentences (clauses) $S$ that contain at least one token in the set of entity types $\mathcal{T}$. Quality meta-patterns are selected by some criteria and further grouped into synonymous groups $M P G=\left[m p_{1}, m p_{2}, \ldots, m p_{k}\right]$, in which $m p_{i}$ and $m p_{j}$ are synonymous meta-patterns.

Candidate meta-pattern generation. We assume the meta-patterns for relationship extraction should contain at least two typed entities. Given the typed clauses from the previous step, we take the segment between the first and last typed entity in each input clause as a candidate meta-pattern. This step generates a large number of noisy meta-patterns. These candidate meta-patterns are then used for quality selection.

\subsection{Quality meta-pattern selection}

Given a large number of candidate meta-patterns, we use a set of contextual features to train a classifier that estimates the quality of each candidate meta-pattern. These contextual features have also been adopted by MetaPAD [20].

(1) Frequency: A quality meta-pattern should occur frequently in the corpus. We use the normalized count of each meta-pattern $c(m p) / N$, where $N$ is the total number of word tokens in the corpus, to measure the frequency of each meta-pattern. We ignore all the meta-patterns that appear less than 10 times in the corpus, i.e., $c(m p)<10$.

(2) Concordance: A quality meta-pattern should have a frequency significantly higher than that is expected due to chance, which 
is a higher concordance. The null hypothesis is: word tokens in the corpus is generated from a series of independent Bernoulli trials. Suppose the total number of word tokens $N$ in the corpus is very large. The expected frequency of a pair of sub-patterns $\left\langle m p_{l}, m p_{r}\right\rangle$ under the null hypothesis is:

$$
\mu_{0}\left(c\left(\left\langle m p_{l}, m p_{r}\right\rangle\right)\right)=N * p\left(m p_{l}\right) * p\left(m p_{r}\right),
$$

where $p(m p)=\frac{c(m p)}{N}$ is the empirical probability of the metapattern $m p$. For each meta-pattern $m p$, we examine all the divisions of dividing $m p$ into $\left\langle m p_{l}, m p_{r}\right\rangle$ without overlapping. We use $\mathrm{Z}$ score to measure the concordance of each meta-pattern $m p$ by finding the division $\left\langle m p_{l}, m p_{r}\right\rangle$ with the maximum $\mathrm{Z}$ score:

$$
Z(m p)=\max _{\left\langle m p_{l}, m p_{r}\right\rangle=m p} \frac{c(m p)-\mu_{0}\left(c\left(\left\langle m p_{l}, m p_{r}\right\rangle\right)\right)}{\left.\sigma_{\left\langle m p_{l}, m p_{r}\right\rangle}\right\rangle},
$$

where $\sigma_{\left\langle m p_{l}, m p_{r}\right\rangle}$ is the standard deviation of the counts of $m p_{l}$ and $m p_{r}$. For example, the meta-pattern "CHEMICAL against CHEMICAL induced DISEASE” may have a higher concordance than the meta-pattern "GENE, GENE and GENE", thus is more likely to be a quality meta-pattern.

(3) Informativeness: A quality meta-pattern should have more informative context words. We use the average inverse document frequency (IDF) score of all the non-type context words $c w(m p)$ in each meta-pattern $m p$ as its informativeness score:

$$
\operatorname{Informativeness}(m p)=\frac{\sum_{w \in c w(m p)} \log \frac{N}{M(w)}}{|c w(m p)|},
$$

where $M(w)$ is the total number of meta-patterns containing the context word $w$. For example, the meta-pattern "CHEMICAL induced GENE" may be more informative than the meta-pattern "CHEMICAL and GENE", thus is more likely to be a quality metapattern.

(4) Completeness: A quality meta-pattern should have a higher ratio between the frequencies of the meta-pattern and its subpatterns. We use the average frequency ratio between each metapattern $m p$ and all its sub-patterns $s u b p(m p)$ as its completeness score:

$$
\text { Completeness }(m p)=\frac{\sum_{s \in s u b p(m p)} \frac{c(m p)}{c(s)}}{|s u b p(m p)|} .
$$

For example, the meta-pattern "CHEMICAL against CHEMICAL induced DISEASE” may be more complete than its sub-pattern "CHEMICAL against", thus is more likely to be a quality metapattern.

(5) Coverage: A quality meta-pattern should extract more distinct instances from the corpus. We used the normalized count of the number of distinct instances $i c(m p) / N$ extracted by each meta-pattern $m p$ to measure the coverage of each meta-pattern.

After extracting the above features for each meta-pattern, we build a random forest based classifier to learn the quality function $Q(m p)$ that maps each meta-pattern to a quality score. We manually selected less than 20 positive and negative meta-patterns, respectively, as the training examples for the model training. After obtaining the quality score of each meta-pattern, we select the meta-pattern with a quality score above a threshold $\delta$ as quality meta-patterns. Here we use $\delta=0.9$. We also assign the same quality score to the extracted instances of each meta-pattern as the instance score for comparison with other OpenIE models.

\subsection{Synonymous meta-pattern grouping}

Synonymous meta-patterns expressing the same kind of relationship should be grouped together to reduce the redundancy of extracted relationship types and enrich the relationship instances under each relationship type. We group synonymous meta-patterns under the following two assumptions:

(1) Synonymous meta-patterns should have the same entity types. For example, "CHEMICAL induces DISEASE" and "CHEMICAL induces GENE" cannot be synonymous meta-patterns.

(2) Synonymous meta-patterns should share similar extracted relationship instances. For example, in Figure 3, the four metapatterns share some extracted instances and all expressing the meaning of "CHEMICAL induces DISEASE", thus should be grouped as a single relationship type.

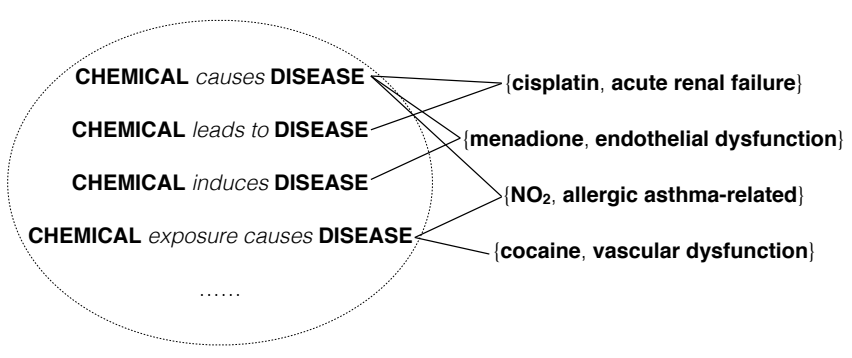

Figure 3: Grouping synonymous patterns by their shared instance sets.

We use a different method for pattern grouping compared with MetaPAD. For each meta-pattern, we generate the term frequencyinverse document frequency (TF-IDF) vector of its instance sets as its feature vector. The instances also contain the type information. Then we perform k-means clustering to group synonymous metapatterns in groups. Since the number of groups cannot be predecided, we set $\mathrm{k}$ to be the total number of quality meta-patterns divided by 5 . We assume that on average, each group will contain 5 synonymous meta-patterns. Examples of synonymous meta-pattern groups are discussed in Section 6.3.

\section{EXPERIMENTS}

\subsection{Dataset}

CPIE only requires biomedical text corpus as input. To test the effectiveness of CPIE, we collect a subset of PubMed paper abstracts. The PubMed id (PMID) of each selected paper can be found in the Comparative Toxicogenomics Database (CTD) [8], in which all the selected PMIDs are shown to contain some biomedical relationships. CTD is a human-curated database containing biological entities and their relationships. The entity and relationship statistics of our collected papers in CTD are shown in Table 2.

We focus on three entity types in CTD: gene, chemical, and disease. Among these three entity types, there are three relation types in CTD: chemical-gene, chemical-disease, and disease-gene relationships. We first randomly select 248,064 relationships from 
Table 2: Statistics of the CTD dataset subset used in our experiments.

\begin{tabular}{c|ccc}
\hline & \# of Entity & \# of Relation & \# of PMID \\
\hline Chemical-Gene & $\begin{array}{c}\text { Chemical: 7,187 } \\
\text { Gene: 667 }\end{array}$ & 163,126 & 26,786 \\
\hline Chemical-Disease & $\begin{array}{c}\text { Chemical: 7,187 } \\
\text { Disease: 598 }\end{array}$ & 84,104 & 1,619 \\
\hline Disease-Gene & $\begin{array}{c}\text { Gene: 669 } \\
\text { Disease: 599 }\end{array}$ & 834 & 1,622 \\
\hline Total & $\begin{array}{c}\text { Chemical: 7,187 } \\
\text { Gene: 669 } \\
\text { Disease: 599 }\end{array}$ & $\mathbf{2 4 8 , 0 6 4}$ & $\mathbf{2 8 , 0 0 7}$ \\
\hline
\end{tabular}

the above three relation types that are associated with experimental evidence in the CTD database. Among these relationships, there are 7,187 chemical, 669 gene and 599 disease entities. CTD also provides the PMIDs of PubMed papers related to these selected relationships. We collect all the 28,007 PubMed abstracts that are shown to be associated with the above relationships in CTD as our input corpus. All the following experiments are performed on this PubMed subset corpus.

\subsection{Performance comparison}

Baselines. To show the effectiveness of CPIE, we compare it with the following state-of-the-art OpenIE approaches:

- ClausIE [10] adopts clause patterns to handle long-distance relationships.

- Stanford OpenIE [2] learns a clause splitter via distant training data.

- Ollie [37] utilizes open pattern learning and extracts patterns over dependency path and part-of-speech tags.

- MinIE [13] refines tuples extracted by ClausIE by identifying and removing parts that are considered overly specific.

Evaluation Metrics. We randomly sample 100 sentences from the 28,007 input PubMed abstracts for performance comparison. All of the compared benchmarks, as well as our method, will assign a confidence score to each extracted tuple. We rank all the tuples according to their confidence scores. Based on the ranking list, the following measures can be adopted: (1) $P @ k$ is the ratio of correct tuples in the top $k$ extractions. (2) MAP is the mean average precision of the whole ranking list. (3) NDCG@k is the normalized discounted cumulative gain at rank $k$. The detailed definition of these common ranking measures can be found in [38]. Note that we do not use recall in OpenIE since it is infeasible to know all the "correct" tuples.

For each method, we select the top 100 tuples from its ranking list and manually label them. The annotator is asked to evaluate without knowing which model produced the results, eliminating potential bias in evaluation. Similar to the settings in previous studies [10], one tuple will be judged as correct if it reads smoothly and meets the fact described in the sentence. For example, both ("DISEASE", "is", "DISEASE") and ("DISEASE", "induced by", "CHEMICAL in SPECIES") are correct. However, ("SPECIES”, "is", "a CHEMICAL") and ("CHEMICAL", "inhibited", "GENE and") will not be counted
Table 3: Performance comparison with state-of-the-art OpenIE systems, using Precision (P@k), Mean Average Precision (MAP) and Normalized Discounted Cumulative Gain (NDCG@k). $k=50,100$.

\begin{tabular}{c|ccccc}
\hline & P@50 & P@100 & MAP & NDCG@50 & NDCG@100 \\
\hline ClausIE [10] & 0.800 & 0.860 & 0.800 & 0.710 & 0.875 \\
Stanford [2] & 0.800 & 0.800 & 0.765 & 0.714 & 0.870 \\
Ollie [37] & 0.920 & 0.890 & 0.917 & 0.935 & 0.982 \\
MinIE [13] & 0.840 & 0.830 & 0.820 & 0.800 & 0.918 \\
CPIE & $\mathbf{0 . 9 4 0}$ & $\mathbf{0 . 9 0 0}$ & $\mathbf{0 . 9 5 6}$ & $\mathbf{0 . 9 5 6}$ & $\mathbf{0 . 9 9 1}$ \\
\hline
\end{tabular}

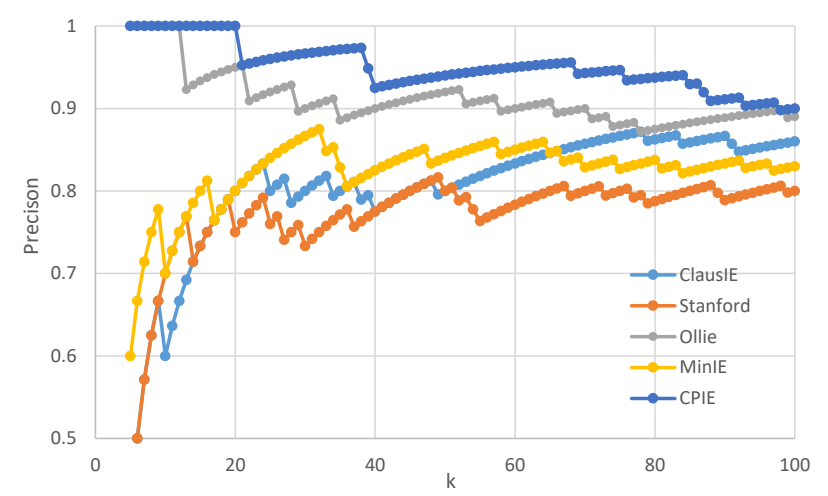

Figure 4: The Precision@ $k$ curves of different methods.

since they have logical or syntactical mistakes. Besides, each tuple should describe exactly one proposition. Tuples with zero or more than two propositions (e.g., "CHEMICAL", "and", "CHEMICAL") and ("DISEASE", "is", "induced by CHEMICAL and has no relationships with GENE”)) will be labeled as incorrect.

Results. Table 3 and Figure 4 demonstrate the performances of different OpenIE approaches. Our method CPIE can be seen as clause extraction + meta-pattern discovery. We also tried MetaPAD alone without clause extraction but it doesn't work due to pattern sparsity in biomedical text. In Table 3, our method is consistently the best according to the rank-based measures, especially compared with ClauseIE without meta-pattern discovery. Ollie ranks the second and achieves a similar $P @ 100$ with our method. However, for MAP and NDCG@50, CPIE outperforms Ollie by a large margin, indicating we have a rather high precision for top-ranked tuples. In Figure 4 , our curve is higher than other baselines for any $k$. Besides, we can observe that for CPIE and Ollie, $P @ k$ decreases with $k$, which means the tuple score is a good indicator of the correctness. From this perspective, the features we defined in Section 5.1 are reliable in evaluating the quality of tuples or meta-patterns.

Distinctiveness and Simplicity. For OpenIE, there are two other important criteria.

- Distinctiveness: For the same sentence, we expect that the extracted tuples should have different semantics with each other. It is not satisfying if they are just paraphrasing each other.

- Simplicity: Each tuple should clearly explain only one proposition. The ideal cases could be using phrases with two or three words to represent subjects, predicates, and objects.

A direct way to examine distinctiveness of our extractions is to calculate the average Jaccard similarity between extractions from 


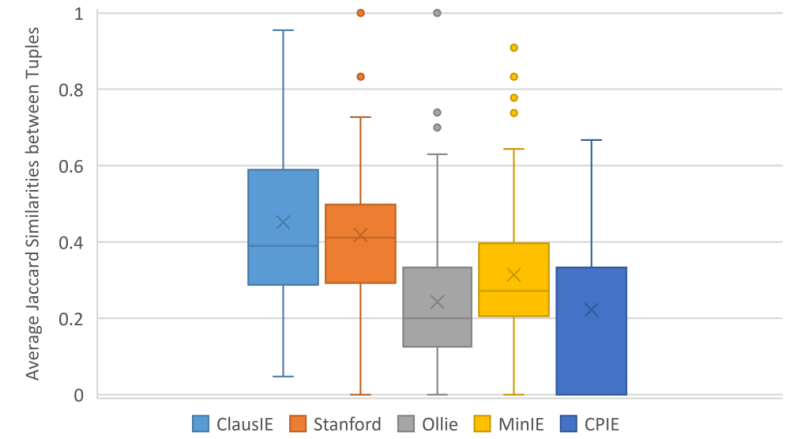

Figure 5: Average Jaccard similarity between extracted tuples in each sentence.

the same sentence. Suppose we get $n$ tuples $\left\{\left(h_{1}, r_{1}, t_{1}\right),\left(h_{2}, r_{2}, t_{2}\right)\right.$, $\left.\ldots,\left(h_{n}, r_{n}, t_{n}\right)\right\}$ from sentence $s^{1}$, the average Jaccard similarity is defined as

$$
\operatorname{Avg} \operatorname{Jacc} \operatorname{Sim}(s)=\frac{1}{\left(\begin{array}{c}
n \\
2
\end{array}\right)} \sum_{1 \leq i<j \leq n} \frac{\left|\left\{h_{i}, r_{i}, t_{i}\right\} \cap\left\{h_{j}, r_{j}, t_{j}\right\}\right|}{\left|\left\{h_{i}, r_{i}, t_{i}\right\} \cup\left\{h_{j}, r_{j}, t_{j}\right\}\right|},
$$

where $\left\{h_{i}, r_{i}, t_{i}\right\}$ denotes the set of tokens appearing in any component of tuple $i$.

We present the average Jaccard similarity distribution of all sentences in Figure 5, from which we can clearly see that Ollie and CPIE extracts the most distinctive facts as they both consider not to be overly specific. In contrast, ClausIE and Stanford OpenIE suffer for the duplication problem.

To evaluate simplicity, we calculate the average tuple length for each sentence:

$$
\operatorname{Avg} \operatorname{Tup} \operatorname{Len}(s)=\frac{1}{n} \sum_{i=1}^{n}\left|\left\{h_{i}, r_{i}, t_{i}\right\}\right|
$$

For different approaches, the distributions of the average tuple length are shown in Figure 6. CPIE again performs the best since meta-patterns are considered to be short and clear. For the tuples extracted by ClausIE, the object component is always very complicated since ClauseIE directly segment the whole sentence into several parts. Sometimes the tuples are too long to illustrate one proposition clearly. According to our evaluation protocol, these tuples will be judged as wrong. In fact, even if the long tuples are logically and syntactically correct, it may not help downstream applications.

\subsection{Case study}

We first look at the quality meta-patterns and the synonymous meta-pattern groups as extracted relation types. Then we examine some extracted relation tuples in detail.

6.3.1 Quality meta-pattern distribution. After candidate metapattern generation, we get 185,403 two-entity and three-entity candidate meta-patterns in total. Most of the candidate meta-patterns appear less than 10 times in the corpus. Those low-frequency metapatterns have the potential to be further resolved and consolidated

\footnotetext{
${ }^{1}$ We only calculate the average Jaccard similarity when $n \geq 2$, and here we only consider 2-ary relations since multi-ary ones may be extensions of them.
}

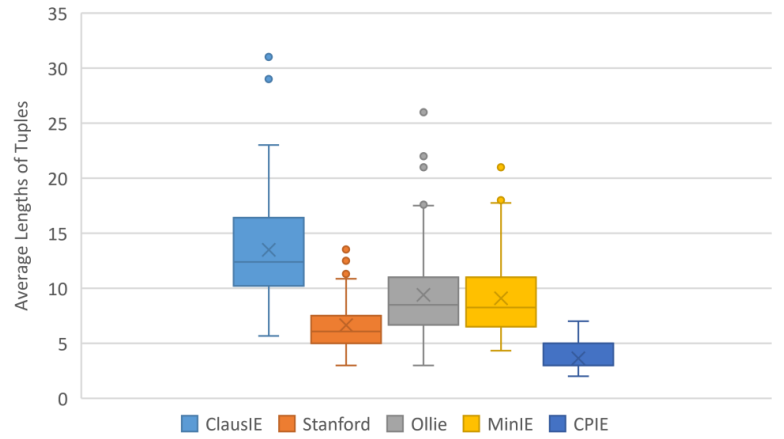

Figure 6: Average length of extracted tuples in each sentence.

with the high-frequency meta-patterns. In this study, we ignore those low-frequency meta-patterns, which results in around 1,000 candidate meta-patterns for quality validation. After ranking these candidate meta-patterns with their quality score (a score between 0 and 1), we selected those meta-patterns with a quality score above 0.9 as quality meta-patterns, which results in around 200 quality meta-patterns.

The count distribution of quality meta-patterns is shown in Figure 7. Different types of the meta-patterns are in different colors in the pie chart. For example, "CHEMICAL" means all the entities in the patterns are chemicals, and "GENE, CHEMICAL" means the pattern consists of both chemicals and genes regardless of their order. From the pie chart, we can see that most of the meta-patterns are relationships between gene and chemical, followed by metapatterns between disease and chemical, and meta-patterns between chemicals.

In Table 4, we list the top 15 quality meta-patterns and their counts. These top meta-patterns describe concrete biomedical relationships between different entity types, which indicates that our quality pattern selection method performs well. The typical raw counts of these quality patterns are around 100 to 300 , which is in the medium range. Apparently, the frequency is not the most indicating feature for quality meta-patterns. Informativeness and coverage play a more important role in identifying quality metapatterns according to our feature importance analysis of the random forest classifier.

6.3.2 Synonymous meta-pattern groups. We group quality metapatterns into around 40 synonymous groups. In Table 5, we list some examples of the synonymous pattern groups. For example, the first group refers to the relation type "CHEMICAL induce DISEASE", which includes meta-patterns such as "CHEMICAL causes DISEASE", "CHEMICAL leads to DISEASE", "CHEMICAL induces DISEASE" and "CHEMICAL exposure causes DISEASE". The metapatterns within each group has very close semantic meaning and each group can be regarded as a specific relation type. These synonymous meta-pattern groups show the effectiveness of CPIE in automatically extracting relation types from massive corpus without supervision. By grouping synonymous meta-patterns together, we reduce the redundancy of extracted relation types and enrich 


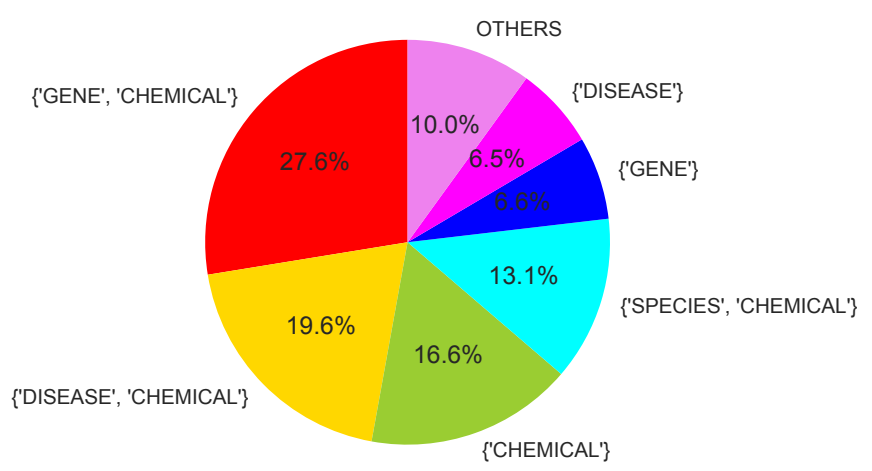

Figure 7: Pie chart of quality meta-pattern counts by group.

Table 4: Top-15 quality meta-patterns.

\begin{tabular}{ll}
\hline Quality meta-patterns & Count \\
\hline CHEMICAL induced DISEASE & 306 \\
CHEMICAL inhibited GENE & 270 \\
CHEMICAL increased GENE & 245 \\
DISEASE be induced by CHEMICAL & 233 \\
DISEASE induced by CHEMICAL & 181 \\
CHEMICAL inhibits GENE & 153 \\
GENE receptor is GENE & 145 \\
GENE inhibitor CHEMICAL & 138 \\
CHEMICAL inhibited CHEMICAL & 126 \\
CHEMICAL inhibited DISEASE & 101 \\
DISEASE be induced by CHEMICAL in SPECIES & 25 \\
CHEMICAL induces apoptosis in SPECIES DISEASE & 25 \\
DISEASE induced by CHEMICAL in SPECIES & 20 \\
CHEMICAL against CHEMICAL induced DISEASE & 15 \\
SPECIES be treated with CHEMICAL & 275
\end{tabular}

the relation instances for each type, which makes the output more structured for downstream applications.

6.3.3 Relationship instances extracted by quality meta-patterns. For each quality meta-pattern we extracted, we perform pattern matching in the input corpus and successfully identify many relation tuples for each meta-pattern. In Table 6, we list some examples of the quality meta-pattern and its corresponding relation tuples, together with the PMID in which we extracted this tuple. We showed that the tuple extraction is of high accuracy in Section 6.2. For example, the first meta-pattern "CHEMICAL increased GENE" extracts a relation tuple ("forskolin", "renin") under this relation type from the Pubmed abstract with PMID "9256163". The original sentence is: "Forskolin (10 microM), an activator of adenylyl cyclase, and terbutaline (100 microM), a beta2-adrenergic agonist known to increase cAMP levels, also increased renin mRNA and prorenin release." Although the two entities, "forskolin" and "renin", are far apart in such a long and complicated sentence, CPIE is able to accurately extract them under the relation of "CHEMICAL increased GENE". One may note that, in this example, "renin mRNA and prorenin release" could be a more accurate subject in the "increased" relation.
It indicates that extending entity recognition to entity phrase recognition for prepossessing may further improve the performance of our system.

Moreover, there are more than one relation type and more than one relation instance in the above sentence. CPIE will also be able to extract the relation tuple ("terbutaline", "renin") under the relation type "CHEMICAL increased GENE", and the relation tuple ("terbutaline", "cAMP") under the relation type "CHEMICAL increased CHEMICAL" simultaneously. This is mainly because we first resolve the sentence structure by extracting short clauses, and then extract quality meta-patterns based on the extracted clauses. This example also shows the power of CPIE in dealing with real-world biomedical literature with complicated sentence structures and rich information in an efficient and high-quality way.

\section{DISCUSSION}

\subsection{Biomedical named entity recognition with distant supervision}

Current meta-patterns only include the most common biomedical entity types - gene, chemical, and disease. Recognizing more entity types will further enrich the extracted meta-patterns and relation tuples. For example, this is a sentence from PubMed papers with PMID "236533": "Colectomy is more effective than high-dose steroid therapy in reversing the growth retardation caused by ulcerative colitis and is of greatest value if not delayed too long." In this sentence, "colectomy" and "steroid therapy" are treatment technologies and "ulcerative colitis" is a disease. If we can recognize the entity type "TREATMENT", we will be able to extract a relation type "TREATMENT treat DISEASE" from the above sentence.

Most biomedical named entity recognition systems use supervised machine learning models, which require human labeled training dataset for model development. However, it may not be possible to acquire the human labeled training data for all the entity types that we are interested in. One way is to leverage distant supervision, which automatically labels the corpus by some distant examples in the knowledge base and then use this partially labeled corpus for model training. For example, Medical Subject Headings (MeSH) is a knowledge base for biomedical entities. We can find "colectomy" in $\mathrm{MeSH}$ ontology, which can serve as a distant supervision example for recognizing the entity type "TREATMENT". It will greatly benefit our current framework if more entity types can be recognized as the first step of the pipeline.

\subsection{Extend meta-pattern extraction}

The current framework utilizes ClausIE to extract short clauses from input sentences and resolve the long and complicated sentence structures. However, the ClausIE output is often noisy and redundant, and errors from ClausIE can be propagated down to the next step of meta-pattern extraction. One way is to directly extend the texture meta-pattern extraction method to long and complicated sentence structures. For example, patterns on the dependencyparsing tree of the sentence can be incorporated with sequential textual patterns for long-distance pattern discovery. How to extend the meta-pattern extraction methods to directly extract meta-patterns on such complicated sentences is an interesting problem. 
Table 5: Examples of synonymous groups of the quality meta-patterns.

\begin{tabular}{ll}
\hline Synonymous group & Meta-patterns \\
\hline CHEMICAL induce DISEASE & CHEMICAL causes DISEASE \\
& CHEMICAL leads to DISEASE \\
& CHEMICAL induces DISEASE \\
& CHEMICAL exposure causes DISEASE \\
\hline CHEMICAL inhibit GENE & CHEMICAL decreased GENE \\
& CHEMICAL decreases GENE \\
& CHEMICAL inhibition of GENE \\
& CHEMICAL suppressed GENE \\
\hline CHEMICAL no effect on GENE & CHEMICAL had no effect on GENE \\
& CHEMICAL did not affect GENE \\
\hline DISEASE induced by CHEMICAL in SPECIES & DISEASE induced by CHEMICAL in SPECIES \\
& DISEASE be induced by CHEMICAL in SPECIES \\
\hline SPECIES treated with CHEMICAL & SPECIES were pretreated with CHEMICAL \\
& SPECIES were administered with CHEMICAL \\
& CHEMICAL treated SPECIES \\
& SPECIES be induced by CHEMICAL \\
& SPECIES were exposed to CHEMICAL \\
\hline
\end{tabular}

Table 6: Examples of relationship instances of the quality meta-patterns.

\begin{tabular}{|c|c|c|c|c|}
\hline Meta-pattern & Entity 1 & Entity 2 & Entity 3 & PMID \\
\hline \multirow[t]{5}{*}{ CHEMICAL increased GENE } & forskolin & renin & - & 9256163 \\
\hline & nicotine & GM-CSF & - & 9606035 \\
\hline & TCDD & TGF-alpha & - & 11309286 \\
\hline & $\mathrm{CP} 461$ & PKG & - & 11602670 \\
\hline & beta-naphthoflavone & CYP1B1 & - & 12843640 \\
\hline \multirow[t]{5}{*}{ CHEMICAL inhibited DISEASE } & rapamycin-FKBP & retinoblastoma & - & 7532117 \\
\hline & bile salts & cholestasis & - & 12644037 \\
\hline & TAS-108 & tumor & - & 15671561 \\
\hline & zinc & cytotoxicity & - & 15922008 \\
\hline & DY-9760e & brain edema & - & 16987238 \\
\hline \multirow[t]{5}{*}{ DISEASE be induced by CHEMICAL in SPECIES } & pulmonary toxicity & nitrofurantoin & rats & 1313237 \\
\hline & colon cancers & $\mathrm{PhIP}$ & rats & 14507667 \\
\hline & metabolic disorders & PFOA & human & 23978341 \\
\hline & carcinogenesis & arsenic & humans & 19524636 \\
\hline & liver injury & carbon tetrachloride & rats & 17173083 \\
\hline \multirow[t]{5}{*}{ CHEMICAL induces apoptosis in SPECIES DISEASE } & arsenic trioxide & human & gastric cancer & 11146441 \\
\hline & flavopiridol & human & leukemia & 11464216 \\
\hline & isoflurane & rat & pheochromocytoma & 18227305 \\
\hline & sodium butyrate & human & hepatoma & 15177505 \\
\hline & butyrate & human & hepatoma & 15177505 \\
\hline \multirow[t]{5}{*}{ CHEMICAL against CHEMICAL induced DISEASE } & dimercaptosuccinic acid & arsenic & toxicity & 15998567 \\
\hline & zinc & cadmium & disorders in bone metabolism & 23726800 \\
\hline & selenium & cadmium & hematological disturbances & 24954678 \\
\hline & resveratrol & cisplatin & testicular damage & 28606469 \\
\hline & erdosteine & acetaminophen & renal toxicity & 16532256 \\
\hline
\end{tabular}

\section{CONCLUSIONS}

We propose a novel framework CPIE: Clause+Pattern-guided Information Extraction that automatically extract both relation type and relation tuples with little supervision. CPIE first resolves the long and complicated sentence structures by clause extraction and then uses texture meta-patterns to extract n-ary tuples with entity type information. Quality meta-patterns are selected and synonymous meta-patterns are grouped to produce more structured output for downstream application. Our method achieves the highest precision in comparison with state-of-the-art OpenIE baselines and keeps the distinctiveness and simplicity of extracted relation tuples. Case studies also show the power of CPIE in effectively dealing with real-world biomedical literature with complicated sentence structures and rich information. Future work to improve our framework includes: (1) include more entity types with distant supervision, (2) replace ClausIE with an extended meta-pattern extraction method to reduce error propagation. 


\section{ACKNOWLEDGMENTS}

The research was sponsored in part by grant 1U54GM114838 awarded by NIGMS through funds provided by the trans-NIH Big Data to Knowledge (BD2K) initiative (www.bd2k.nih.gov), U.S. Army Research Lab. under Cooperative Agreement No. W911NF-09-2-0053 (NSCTA), DARPA under Agreement No. W911NF-17-C-0099, National Science Foundation IIS 16-18481, IIS 17-04532, and IIS-1741317, and DTRA HDTRA11810026. Any opinions, findings, and conclusions or recommendations expressed in this document are those of the author(s) and should not be interpreted as the views of any U.S. Government. The U.S. Government is authorized to reproduce and distribute reprints for Government purposes notwithstanding any copyright notation hereon.

\section{REFERENCES}

[1] Sophia Ananiadou, Paul Thompson, Raheel Nawaz, John McNaught, and Douglas B Kell. 2014. Event-based text mining for biology and functional genomics. Briefings in functional genomics 14, 3 (2014), 213-230.

[2] Gabor Angeli, Melvin Jose Johnson Premkumar, and Christopher D Manning. 2015. Leveraging linguistic structure for open domain information extraction. In ACL'15. ACL, 344-354.

[3] Michele Banko, Michael J Cafarella, Stephen Soderland, Matthew Broadhead, and Oren Etzioni. 2007. Open information extraction from the web.. In IFCAI'07. AAAI, 2670-2676.

[4] Yonggang Cao, Feifan Liu, Pippa Simpson, Lamont Antieau, Andrew Bennett, James J Cimino, John Ely, and Hong Yu. 2011. AskHERMES: An online question answering system for complex clinical questions. Fournal of biomedical informatics 44, 2 (2011), 277-288.

[5] Wendy W Chapman and K Bretonnel Cohen. 2009. Guest Editorial: Current issues in biomedical text mining and natural language processing. Fournal of biomedical informatics 42, 5 (2009), 757-759.

[6] Aaron M Cohen and William R Hersh. 2005. A survey of current work in biomedical text mining. Briefings in bioinformatics 6, 1 (2005), 57-71.

[7] Kevin Bretonnel Cohen and Dina Demner-Fushman. 2014. Biomedical natural language processing. Vol. 11. John Benjamins Publishing Company.

[8] Allan Peter Davis, Cynthia J Grondin, Robin J Johnson, Daniela Sciaky, Benjamin L King, Roy McMorran, Jolene Wiegers, Thomas C Wiegers, and Carolyn J Mattingly. 2016. The comparative toxicogenomics database: update 2017. Nucleic acids research 45, D1 (2016), D972-D978.

[9] Nisansa de Silva, Dejing Dou, and Jingshan Huang. 2017. Discovering inconsistencies in pubmed abstracts through ontology-based information extraction. In ACM-BCB'17. ACM, 362-371.

[10] Luciano Del Corro and Rainer Gemulla. 2013. Clausie: clause-based open information extraction. In WWW'13. ACM, 355-366.

[11] Anthony Fader, Stephen Soderland, and Oren Etzioni. 2011. Identifying relations for open information extraction. In EMNLP'11. ACL, 1535-1545.

[12] Carol Friedman, George Hripcsak, Lyuda Shagina, and Hongfang Liu. 1999. Representing information in patient reports using natural language processing and the extensible markup language. Fournal of the American Medical Informatics Association 6, 1 (1999), 76-87.

[13] Kiril Gashteovski, Rainer Gemulla, and Luciano Del Corro. 2017. MinIE: minimizing facts in open information extraction. In EMNLP'17. ACL, 2630-2640.

[14] Ralph Grishman. 2012. Information Extraction: Capabilities and Challenges.(2012). Notes prepared for the 2012 International Winter School in Language and Speech Technologies (2012).

[15] Jinghang Gu, Fuqing Sun, Longhua Qian, and Guodong Zhou. 2017. Chemicalinduced disease relation extraction via convolutional neural network. Database 2017 (2017).

[16] Udo Hahn, K Bretonnel Cohen, Yael Garten, and Nigam H Shah. 2012. Mining the pharmacogenomics literature-a survey of the state of the art. Briefings in bioinformatics 13, 4 (2012), 460-494.

[17] Rave Harpaz, Alison Callahan, Suzanne Tamang, Yen Low, David Odgers, Sam Finlayson, Kenneth Jung, Paea LePendu, and Nigam H Shah. 2014. Text mining for adverse drug events: the promise, challenges, and state of the art. Drug safety 37, 10 (2014), 777-790.

[18] Marti A Hearst. 1992. Automatic acquisition of hyponyms from large text corpora. In ACL'92. ACL, 539-545.

[19] Jing Jiang. 2012. Information extraction from text. In Mining text data, Charu C Aggarwal and ChengXiang Zhai (Eds.). Springer, 11-41.

[20] Meng Jiang, Jingbo Shang, Taylor Cassidy, Xiang Ren, Lance M Kaplan, Timothy P Hanratty, and Jiawei Han. 2017. MetaPAD: Meta Pattern Discovery from Massive Text Corpora. In KDD'17. ACM, 877-886.
[21] Dan Klein and Christopher D Manning. 2003. Accurate unlexicalized parsing. In ACL'03.

[22] Martin Krallinger, Alfonso Valencia, and Lynette Hirschman. 2008. Linking genes to literature: text mining, information extraction, and retrieval applications for biology. Genome biology 9, 2 (2008), S8.

[23] Qi Li, Meng Jiang, Xikun Zhang, Meng Qu, and Jiawei Han. 2018. TruePIE: Discovering reliable patterns in pattern-based information extraction. In KDD'18. ACM.

[24] Zhiheng Li, Zhihao Yang, Hongfei Lin, Jian Wang, Yingyi Gui, Yin Zhang, and Lei Wang. 2016. CIDExtractor: A chemical-induced disease relation extraction system for biomedical literature. In BIBM'16. IEEE, 994-1001.

[25] Feifan Liu, Jinying Chen, Abhyuday Jagannatha, and Hong Yu. 2016. Learning for biomedical information extraction: Methodological review of recent advances. arXiv preprint arXiv:1606.07993 (2016).

[26] Stéphane M Meystre, Guergana K Savova, Karin C Kipper-Schuler, John F Hurdle, et al. 2008. Extracting information from textual documents in the electronic health record: a review of recent research. Yearb Med Inform 35, 8 (2008), 128-144.

[27] Tom M Mitchell, William W Cohen, Estevam R Hruschka Jr, Partha Pratim Talukdar, Justin Betteridge, Andrew Carlson, Bhavana Dalvi Mishra, Matthew Gardner, Bryan Kisiel, Jayant Krishnamurthy, et al. 2015. Never Ending Learning.. In $A A A I^{\prime} 15$. AAAI, 2302-2310.

[28] Dana Movshovitz-Attias and William W Cohen. 2012. Bootstrapping biomedical ontologies for scientific text using nell. In Proceedings of the 2012 Workshop on Biomedical Natural Language Processing. ACL, 11-19.

[29] Ndapandula Nakashole, Gerhard Weikum, and Fabian Suchanek. 2012. PATTY: a taxonomy of relational patterns with semantic types. In EMNLP'12. ACL, 11351145 .

[30] Victoria Nebot and Rafael Berlanga. 2011. Semantics-aware open information extraction in the biomedical domain. In Proceedings of the 4th International Workshop on Semantic Web Applications and Tools for the Life Sciences. ACM, 84-91.

[31] Victoria Nebot and Rafael Berlanga. 2014. Exploiting semantic annotations for open information extraction: an experience in the biomedical domain. Knowledge and information Systems 38, 2 (2014), 365-389.

[32] Alexander Nikitin, Sergei Egorov, Nikolai Daraselia, and Ilya Mazo. 2003. Pathway studio - the analysis and navigation of molecular networks. Bioinformatics 19 , 16 (2003), 2155-2157.

[33] Yifan Peng, Chih-Hsuan Wei, and Zhiyong Lu. 2016. Improving chemical disease relation extraction with rich features and weakly labeled data. fournal of cheminformatics 8,1 (2016), 53.

[34] Jakub Piskorski and Roman Yangarber. 2013. Information extraction: Past, present and future. In Multi-source, multilingual information extraction and summarization, Thierry Poibeau, Horacio Saggion, Jakub Piskorski, and Roman Yangarber (Eds.). Springer, 23-49.

[35] Dietrich Rebholz-Schuhmann, Anika Oellrich, and Robert Hoehndorf. 2012. Textmining solutions for biomedical research: enabling integrative biology. Nature Reviews Genetics 13, 12 (2012), 829.

[36] Fabio Rinaldi, Simon Clematide, Hernani Marques, Tilia Ellendorff, Martin Romacker, and Raul Rodriguez-Esteban. 2014. OntoGene web services for biomedical text mining. BMC bioinformatics 15, 14 (2014), S6.

[37] Michael Schmitz, Robert Bart, Stephen Soderland, Oren Etzioni, et al. 2012. Open language learning for information extraction. In EMNLP'12. ACL, 523-534.

[38] Hinrich Schütze, Christopher D Manning, and Prabhakar Raghavan. 2008. Introduction to information retrieval. Vol. 39. Cambridge University Press.

[39] Matthew S Simpson and Dina Demner-Fushman. 2012. Biomedical text mining: A survey of recent progress. In Mining text data, Charu C Aggarwal and ChengXiang Zhai (Eds.). Springer, 465-517.

[40] Chih-Hsuan Wei, Hung-Yu Kao, and Zhiyong Lu. 2013. PubTator: a web-based text mining tool for assisting biocuration. Nucleic acids research 41, W1 (2013), W518-W522.

[41] Chih-Hsuan Wei, Yifan Peng, Robert Leaman, Allan Peter Davis, Carolyn J Mattingly, Jiao Li, Thomas C Wiegers, and Zhiyong Lu. 2015. Overview of the BioCreative V chemical disease relation (CDR) task. In Proceedings of the fifth BioCreative challenge evaluation workshop. Sevilla Spain, 154-166.

[42] Thomas C Wiegers, Allan Peter Davis, and Carolyn J Mattingly. 2014. Web services-based text-mining demonstrates broad impacts for interoperability and process simplification. Database 2014 (2014).

[43] Deyu Zhou, Dayou Zhong, and Yulan He. 2014. Biomedical relation extraction: from binary to complex. Computational and mathematical methods in medicine 2014 (2014), 1-18.

[44] Qi Zhu, Xiang Ren, Jingbo Shang, Yu Zhang, Frank F Xu, and Jiawei Han. 2018. Open Information Extraction with Global Structure Constraints. In $W W W^{\prime} 18$. ACM, 57-58.

[45] Pierre Zweigenbaum, Dina Demner-Fushman, Hong Yu, and Kevin B Cohen. 2007. Frontiers of biomedical text mining: current progress. Briefings in bioinformatics 8,5 (2007), 358-375. 